\title{
Protective Effects of Propolis on Gamma- Irradiated Nigella sativa Extract Induced Blood and Immune Changes in Wistar Rats
}

\author{
${ }^{1,3}$ Osama Moseilhy Saleh, ${ }^{2,5}$ Mohamed Mohamed Soliman, \\ ${ }^{1,4}$ Ahmed Abdel-Khalik Mansour and ${ }^{5}$ Omniya Mahmoud Abdel-Hamid \\ ${ }^{1}$ Department of Medical Biotechnology, Faculty of Applied Medical Sciences, Turabah, Taif University, KSA \\ ${ }^{2}$ Department of Medical Laboratories, Faculty of Applied Medical Sciences, Turabah, Taif University, KSA \\ ${ }^{3}$ Department of Natural Products Research, \\ National Centre for Radiation Research and Technology (NCRRT), Cairo, Egypt \\ ${ }^{4}$ Department of Genetics, Faculty of Agriculture, Ain Shams University, Cairo, Egypt \\ ${ }^{5}$ Department of Biochemistry, Faculty of Veterinary Medicine, Benha University, Kalubiya, Egypt
}

Received 2013-04-29, Revised 2013-05-10; Accepted 2013-05-20

\begin{abstract}
The present study conducted to test the effect of Nigella Sativa (NS, $5 \mathrm{mg} \mathrm{kg}^{-1}$ of body weight), or $\gamma$ irradiated Nigella Sativa (GRNS) on the changes of blood component profiles, liver, kidney functions and immune cytokines secretion in male Wistar rats. Moreover, the possible protection by propolis $\left(200 \mathrm{mg} \mathrm{kg}^{-1}\right.$ B. W.) on the changes induced by NS and GRNS was examined. Results revealed that both NS and GRNS administration for two weeks induced changes in blood, GPT, GOT and urea levels and co-administration with propolis significantly ameliorated such changes. Also, liver histology showed numerous vacuolar degeneration and fatty changes in $\gamma$-irradiated groups which disappeared in presence of propolis. Kidney histology of NS administered rats showed less lymphocytic infiltration, while GRNS groups showed desquamation in the cytoplasm of the renal tubules, hemorrhage in the renal corpuscle and lymphocytic infiltration which disappeared when propolis given together with GRNS. Finally propolis induced protective effect on the changes induced in TNF- $\alpha$ and IL-10 secretion by either NS or GRNS in Wistar rats. In conclusion, the findings of present study clarified the protective effect of propolis on changes induced by $\gamma$ irradiated NS on blood, liver, kidney and cytokines changes in Wistar rats.
\end{abstract}

Keywords: Blood Changes, $\gamma$-Irradiation, Nigella Sativa, Propolis, Wistar Rats

\section{INTRODUCTION}

During last decade the medical applications of radiation technology have been increased rapidly due to development of new diagnostics and therapeutic methods using gamma irradiation in medical therapy (Hendee, 1992). Gamma irradiation treatment increases the annual dose received by both the patients and physicians because of regular exposure to radiation or radio-nuclides intake. Therefore, studying the biological damage induced by ionizing radiation is necessary in assessment of maximum absorbed dose during radiotherapy or diagnosis. Moreover, development of protective agents presented new solutions for recovery of undesired tissue damage induced by ionizing radiation (Borek, 2004).

The ionizing radiations cause damage of the cells directly by ionization of DNA and other cellular targets and indirectly through Reactive Oxygen Species (ROS) (Borek, 2004). Ionizing radiation produces oxygen free radicals in tissues as superoxide anion radicals and other Corresponding Author: Osama Moseilhy Saleh, Medical Biotechnology Department, Faculty of Applied Medical Sciences, Turabah, Taif University, KSA 
oxidants as hydrogen peroxide. It has been shown that radiotherapy causes killing of tumor cells but its usage causes threatening for the integrity and survival of surrounding normal cells (Konopacka and Rogolinski, 2004). Now days, there is increase attention in usage of radiation in diagnosis of diseases. However, exposure to medical irradiation has grown in the past two decades and still increasing to date. The biohazards of irradiation appear from its cumulative levels in body that leads to cancer, cell death, genetic damage and tissue pathology (Borek, 2004). Irradiation causes DNA strand breaking (Eric and Giaccia, 2012). Lymphokines are molecules that mediate molecular pathways common to ionizing irradiation induced apoptosis (Epperly et al., 2001; 2003a). Irradiation causes activation of caspase-3 by DNA fragmentation (Epperly et al., 2002). These pathways are common in apoptosis induction by TNF- $\alpha$ and mediation of other cytokines as IL-1 and IL-8 (Epperly et al., 2003a). The diversity of irradiation used in Medicine, Industry, Agriculture, scientific research and military process increases and protection against irradiation is an important issue (Epperly et al., 2003b; Pearce et al., 2001). Nigella sativa is commonly known as black seed or black cumin and used as an herbal plant all over the world for prevention of various diseases as asthma, hypertension, diabetes, inflammation, cough, bronchitis, headache, eczema, fever, dizziness and influenza (Salem, 2005). The seeds or its oil used as a carminative, diuretics, lactagouge and vermifuge. NS is used in food preparation as a source of spicy and a condiment (Ali and Blunden, 2003). It has codeine like effect as anti-tussive factor (Boskabady et al., 2004). Its water extract contain thymoquinone and oily substances called monoterpenes (El-Tahir et al., 1993). Both Houghton et al. (1995) and Worthen et al. (1998), reported anti-cancer and oxidant activity for NS.

For centuries, beekeepers assumed that bees sealed the beehive with propolis to protect the colony from the elements, such as rain and cold winter drafts and as protector for the hive from diseases and parasites that may attack bees (Gregory et al., 2002). For human health propolis has various functions among which relief inflammation, viral diseases, ulcers, superficial burns or scalds and anti-microbial agents (Gregory et al., 2002). Also propolis has an emollient, immune-modulator, a dental anti-plaque, an anti-tumor growth and a radioprotective factor (Ansorge et al., 2003; Benkovic et al., 2009; Koo et al., 2002; Orsi et al., 2005; Wu et al., 2011). So, the present study aimed to test the effect of normal and $\gamma$-irradiated NS on alterations in cytoimmune-modulatory function and histopathology of liver and kidney in Wistar rats and possible effects of coadministration of propolis together with NS.

\section{MATERIALS AND METHODS}

\subsection{Irradiation Treatment}

Nigella Sativa seeds were purchased from local Egyptian market. Half of the seeds were irradiated with 10 Gy of gamma radiations using caesium 137 units in the National Centre of Radiation Research and Technology (NCRRT), Nasr City, Cairo, Egypt.

\subsection{Nigella Sativa (NS) Extract Preparation}

All seeds (irradiated and non-irradiated) were dried and crushed to a powder with an electronic microniser. 20 $\mathrm{g}$ of powder was dissolved in $200 \mathrm{~mL}$ phosphate buffer saline ( $\mathrm{pH} \mathrm{6.4)}$ and centrifuged at $1000 \mathrm{rpm}$ for $30 \mathrm{~min}$ at $4^{\circ} \mathrm{C}$ to remove residual solid debris. The clear supernatant was collected as soluble extract after removal of the oily layer and insoluble pellet. Protein concentration was determined by Lowry et al. (1951) method.

\subsection{Propolis Extract Preparation}

Propolis $(50 \mathrm{~g})$ was dissolved in $100 \mathrm{~mL}$ diethyl ether with continuous shaking for $48 \mathrm{~h}$ then filtered in Wattmman paper and filtrate was again dissolved in 50 $\mathrm{mL}$ diethyl ether for another $48 \mathrm{~h}$ then final filtrate was dissolved in $50 \mathrm{~mL}$ Distilled water. The final concentration was adjusted to be $200 \mathrm{mg} \mathrm{mL}^{-1}$ by protein assay method.

\subsection{Experimental Design}

Thirty eight weeks old of Wistar rats (200-250 g) were used for this experiment. After one week accommodation, rats were divided into six groups (5 rats per group). The first group used as a negative control. The second group received non-irradiated Nigella sativa extract (NS group) in a dose of $5 \mathrm{mg} \mathrm{kg}^{-1}$ of B.W. orally for two weeks. The third group received orally gamma irradiated NS extract (GRNS) in a dose of (5 mg kg-1 of B.W.) The fourth group received propolis (P group) at a dose of $200 \mathrm{mg} \mathrm{kg}^{-1}$ of B.W. for two weeks. Fifth group received normal NS extract for two weeks followed by propolis together with NS for other two weeks (NSP group). The sixth group received $\gamma$-irradiated NS extract for two weeks then $\gamma$-irradiated NS with propolis for other two weeks (GRNSP). At the end of the experiment, rats were killed by decapitation and plasma was collected for blood and cytokines measurements and assays. Liver and kidney tissues were collected in formalin for histopathology. 


\subsection{Blood Chemistry Analysis}

Plasma measurement for Complete Blood Picture (CBP) was measured using the commercial available kits (Mabaret $\mathrm{Al}$ asafra Lab, Alex. Eypt). GPT, GOT, Urea and creatinine were analyzed spectrophotometrically using kits imported by Clini lab, Giza, Egypt.

\subsection{Histo-Pathological Studies on Kidney and Liver Tissues}

Tissue samples from liver, spleen and kidney were fixed in $10 \%$ buffered formalin and embedded in paraffin. Tissue sections $(5 \mu \mathrm{m})$ were stained with hematoxylin-eosin to study their micro architecture by light microscopy.

\subsection{Cytokines Assays}

Elisa kits for rat IL-1 $\beta$, TNF- $\alpha$ and IL-10 was purchased from Assaypro LLC, 30 Triad South Drive, St. Charles, MO 63301, USA. The measurements were done according to the catalogue instruction guidelines.

\subsection{Statistical Analysis}

Results are expressed as means \pm S.E. of independent experiments. Statistical analysis was done using ANOVA and Fischer's post hoc test, with $\mathrm{p}<0.05$ being considered as statistically significant.

\section{RESULTS}

\subsection{Alterations in Blood Profiles after NS, GRNS and Propolis Administration}

To test the changes induced by administration of either NS or GRNS alone or in combination with propolis for extra two weeks. Changes in blood parameters, liver and kidney function parameters have been measured. As seen in Table 1, propolis in combination with GRNS didn't induce any significant changes in the increase in $\mathrm{Hb}$ concentration and red blood cells count induced by GRNS alone. Unlike Hb and red blood cell results, NS treatment showed significantly increasing in white blood cell counts, while propolis $(\mathrm{p}<0 / 05)$ significantly decreased such alteration. Moreover, GRNS increased insignificantly white blood cell count but administration of propolis together with GRNS normalized such changes. Parallel to that changes, is platelets findings, GRNS significantly decreases platelets number and addition of propolis normalized it to normal levels. Regarding changes in GPT and GOT levels, Table 1 shows that $\gamma$-irradiation treatment induced increasing in GPT and GOT levels and addition of propolis induced normalization to control levels. No significant alterations for NS alone or in combination with propolis on GPT and GOT levels. Finally, we tested the effect of propolis on GRNS induced changes in creatinine and urea levels. As seen in Table 1, propolis has urea lowering effect on GRNS increased urea levels and addition of propolis normalized it and same occurred for propolis when added to NS.

\subsection{Liver and Kidney Histopathology after NS, GRNS and Propolis Administration in Wistar Rats}

\subsubsection{Liver Findings}

The liver of normal control group is lined by hepatic cords of one cell thickness with eosinophilic cytoplasm and centrally located basophilic nuclei around central vein (Fig. 1A). The liver of rats administered NS, showed normal structure (Fig. 1B). While the liver of rats administered with GRNS showed numerous vacuolar degeneration and fatty changes (Fig. 1C). The liver treated with propolis, showed little hydropic degeneration (Fig. 1D). While the liver of rats administered with NS and Propolis (NSP) showed vacuolar degeneration (Fig. 1E).

Table 1. Changes in Blood components profiles, liver and kidney function tests after oral administration of NS, GRNS alone or in combination with propolis for two weeks in Wistar rats

\begin{tabular}{|c|c|c|c|c|c|c|}
\hline Groups parameter & Control & NS & GRNS & Propolis & NSP & GRNSP \\
\hline Hemoglobin (gm/dL) & $12 \pm 0.7$ & $12.5 \pm 0.1$ & $14.1 \pm 0.4^{*}$ & $16.3 \pm 0.7^{*}$ & $14.3 \pm 0.5^{\#}$ & $14.1 \pm 0.5$ \\
\hline R.B.ces $\left(10^{6} / \mathrm{mm}^{3}\right)$ & $4.2 \pm 0.2$ & $4.2 \pm 1.00$ & $5 \pm 0.2^{*}$ & $5.6 \pm 0.2^{*}$ & $4.9 \pm 0.2$ & $4.8 \pm 0.2$ \\
\hline W.B.ces $\left(10^{3} / \mathrm{mm}^{3}\right)$ & $9.4 \pm 0.3$ & $13.6 \pm 0.5^{*}$ & $10.3 \pm 0.6$ & $6.4 \pm 0.1^{*}$ & $7.6 \pm 0.3^{\#}$ & $8.2 \pm 0.5^{\$}$ \\
\hline Platelets $\left(10^{3} / \mathrm{mm}^{3}\right)$ & $331.3 \pm 31.3$ & $283.7 \pm 21$ & $192 \pm 2^{*}$ & $287.7 \pm 1.9$ & $301.3 \pm 18^{\#}$ & $285 \pm 23^{\$}$ \\
\hline GPT (U/L) & $52.4 \pm 11$ & $94.6 \pm 7.3^{*}$ & $99 \pm 16.6^{*}$ & $77 \pm 4.3$ & $97.6 \pm 17.2$ & $84 \pm 6^{\$}$ \\
\hline GOT(U/L) & $68 \pm 10.4$ & $75 \pm 10.4$ & $122 \pm 17.8^{*}$ & $75 \pm 6.4$ & $77 \pm 5.12$ & $73 \pm 10.3^{\$}$ \\
\hline Creatinine $(\mathrm{mg} / \mathrm{dL})$ & $0.8 \pm 0.2$ & $1.1 \pm 0.1$ & $1.1 \pm 0.1$ & $0.9 \pm 0.1$ & $0.9 \pm 0.1$ & $0.8 \pm 0.1$ \\
\hline Urea $(\mathrm{mg} / \mathrm{dL})$ & $39.3 \pm 2.2$ & $36.8 \pm 2.4$ & $59 \pm 7.4^{*}$ & $30.4 \pm 3.8$ & $26.5 \pm 2.3^{\#}$ & $36 \pm 4.1^{\$}$ \\
\hline
\end{tabular}

Serum changes in blood parameters after administration of Nigella Sativa (NS) or Gamma Radiated Nigella Sativa (GRNS) and prior protection in combination with propolis. Hb, hemoglobin; Red Blood Cells (RBCs); White Blood Cells (WBCs); GPT, glutamate pyruvate transaminase; GOT, glutamate oxalacetate transaminase. Values are means \pm S.E for 5 different rats per each group. *; $\mathrm{p}<0.05$ Vs control; ${ }^{\#} \mathrm{p}<0.05$ Vs NS; ${ }^{\$} \mathrm{p}<0.05$ Vs GRNS 

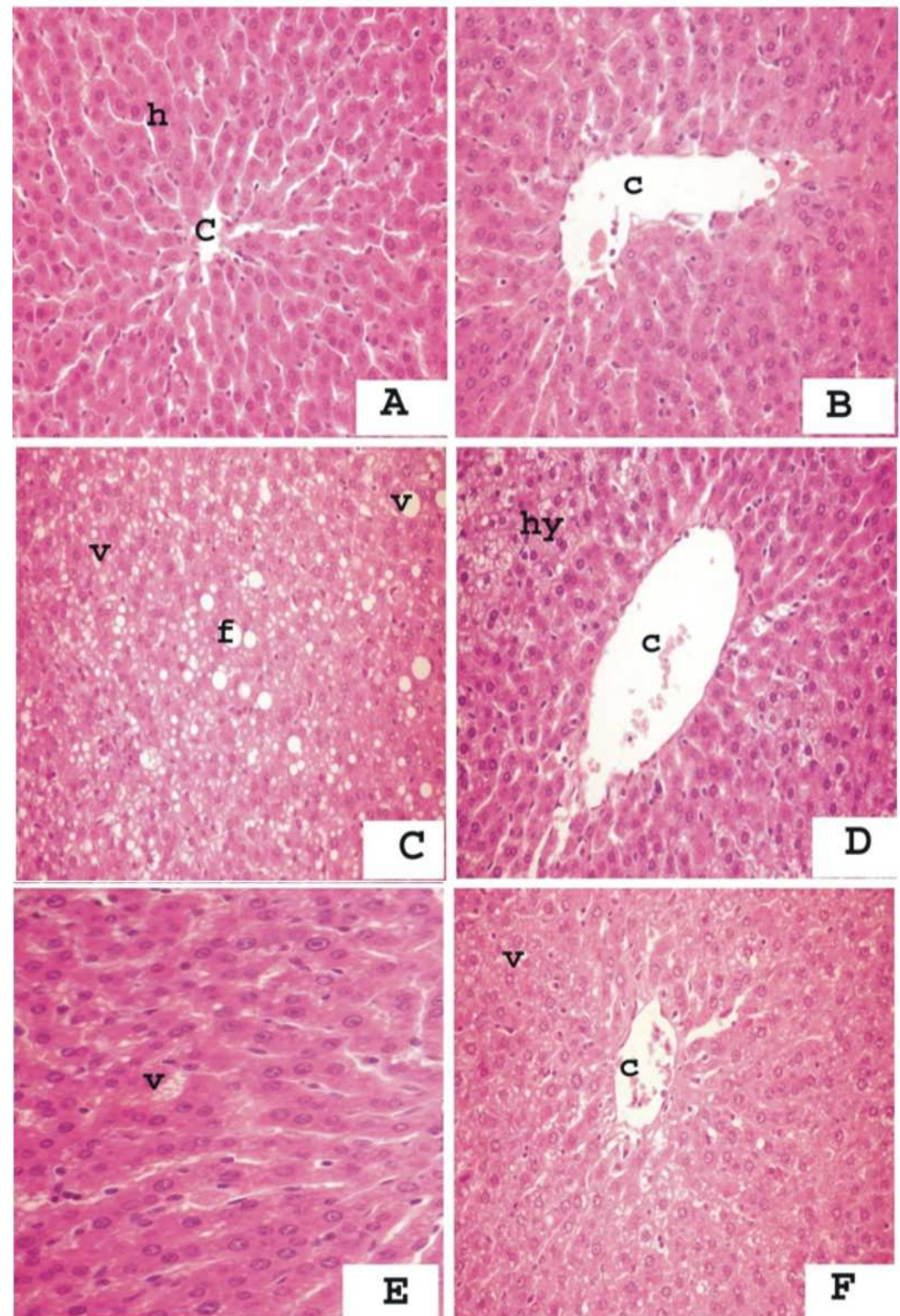

Fig. 1. Protective effect of propolis on NS and GRNS induced changes in Liver histology. Liver histology was examined by H and $E$ stain (A and B) show normal structure of hepatic cord (h) and central vein (c) (C) ( $\gamma$-irradiated NS group) showed numerous vacuolar degeneration (V) and fatty changes (f) (D) (propolis group) shows little hydropic degeneration (hy) and normal central vein (c). (E) (NSP; NS plus propolis) showed vacuolar degeneration (v). (F) (GRNSP, gamma-radiated NS plus propolis) showed disappearance of the fatty changes and very little vacuolar degeneration (v). Original magnification: X400 (A-F) 

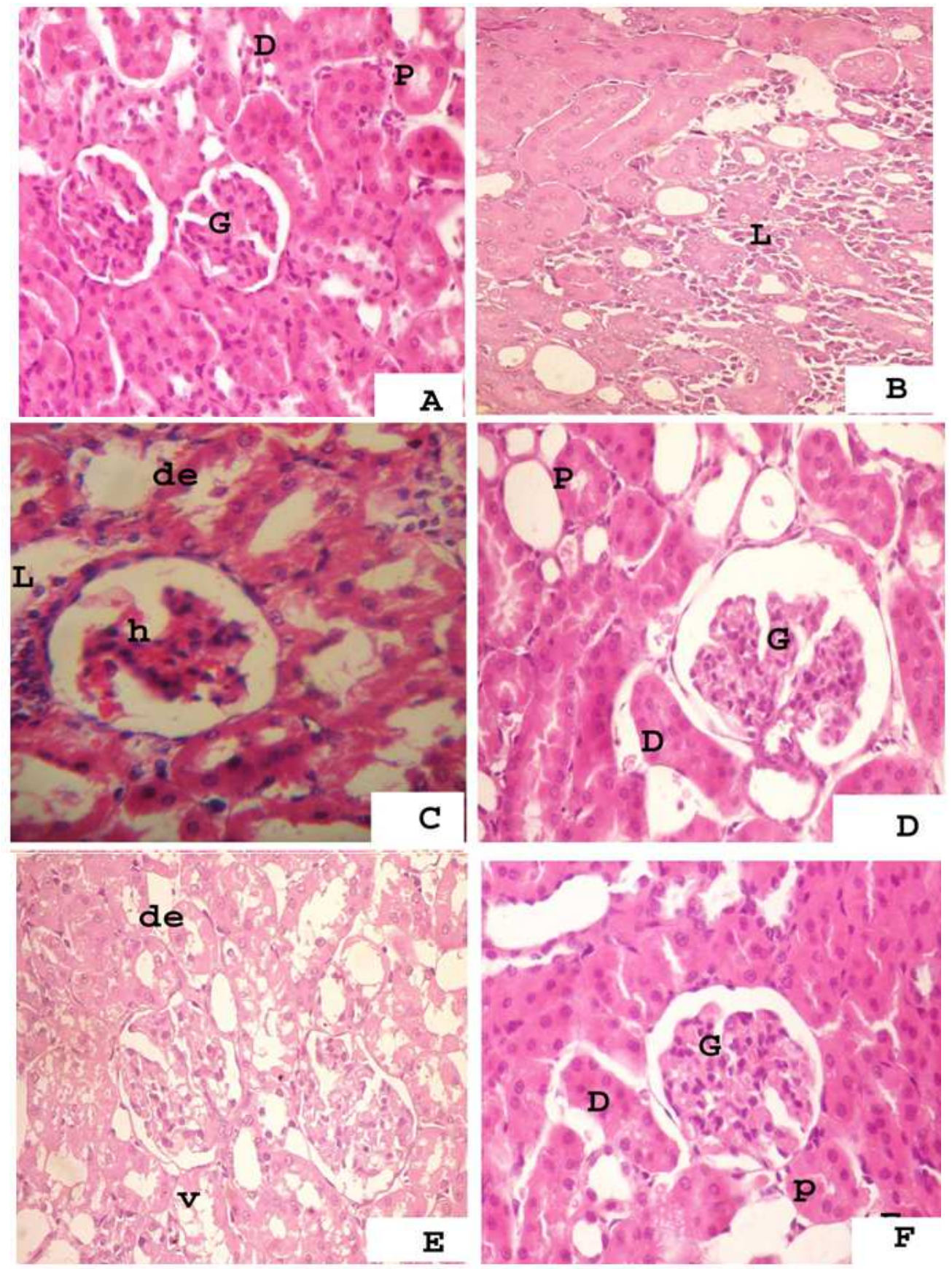

Fig. 2. Protective effect of propolis on NS and GRNS induced changes in kidney histology. Kidney histology was examined by H and E stain. (A) (control) and D (propolis) show normal kidney structure of glomeruli (G), proximal (P) and distal (D) convoluted tubules. (B) shows little lymphocytic infiltration $(\mathrm{L})(\mathrm{C})(\gamma$-irradiated NS group) shows desquamation in the cytoplasm (de) of renal tubules, hemorrhage (h) in renal corpuscles and lymphocytic infiltration (L). (E) (NSP; NS plus propolis) showed vacuolation (v) and desquamation (de) in the cytoplasm of the renal tubules. (F) (GRNSP, gamma-radiated NS plus propolis) showed disappearance of the lymphocytic infiltration and desquamation in the epithelium of the renal tubules. Original magnification: X400 (A-F) 


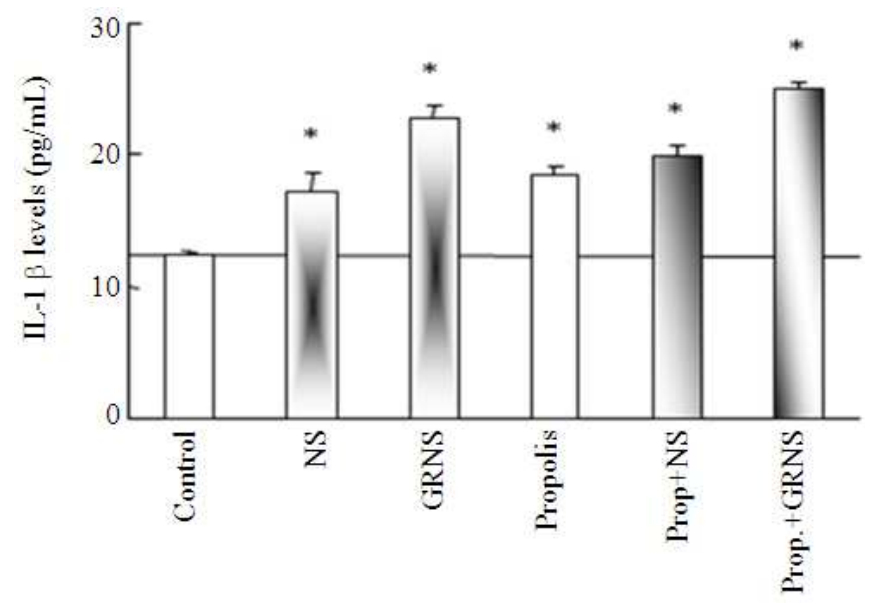

Fig. 3. Effects of propolis on NS and GRNS induced changes in IL-1 $\beta$ levels. Wistar rats were NS, GRNS alone or in combination with propolis for two weeks. Serum levels of IL- $1 \beta$ were measured using ELISA. Values are means \pm S.E.M for 5 different rats for each experiment. ${ }^{*} \mathrm{p}<0.05$ vs. control

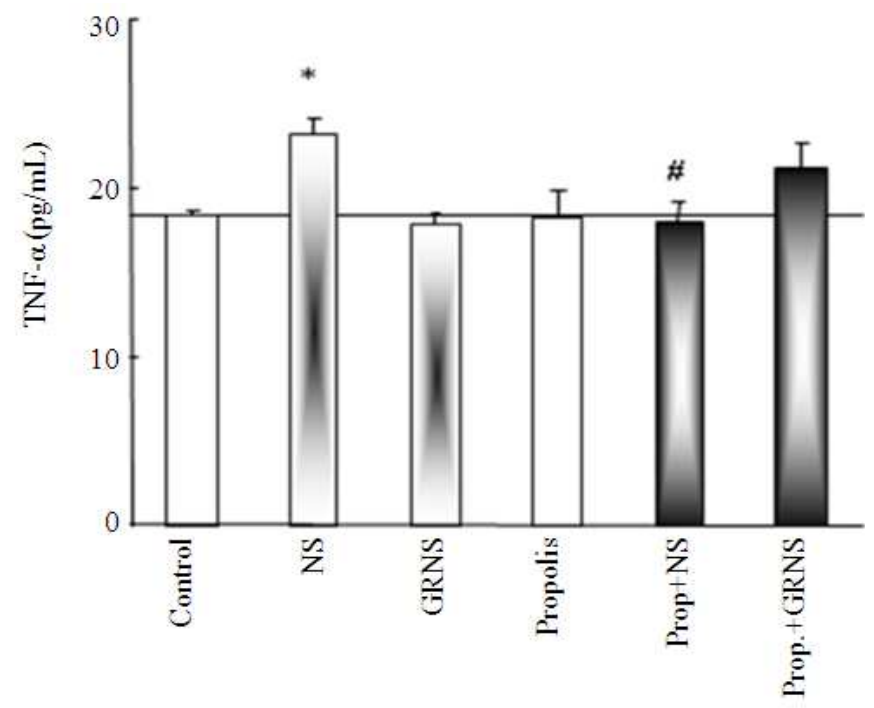

Fig. 4. Protective effects of propolis on NS and GRNS induced changes in TNF- $\alpha$ levels. Wistar rats were NS, GRNS alone or in combination with propolis for 2 weeks. Serum levels of TNF- $\alpha$ were measured using ELISA. Values are means \pm S.E.M for 5 different rats for each experiment. ${ }^{*} \mathrm{p}<0.05$ vs. control and $\# \mathrm{p}<0.05$ Vs. NS group

The liver of rats given GRNS and propolis (GRNSP) showed disappearance of the fatty changes and very little vacuolar degeneration still resident (Fig. 1F).

\subsection{Kidney Findings}

The kidney renal corpuscle lined by simple squamous epithelium. Proximal convoluted tubules lined by pyramidal cells with eosinophilic cytoplasm. While the distal convoluted tubules lined by simple cuboidal epithelium with light eosinophilic cytoplasm and basophilic centrally located nuclei (Fig. 2A). The kidney of rats administered NS, showed lymphocytic infiltration in the parenchyma of the renal tissues (Fig. 2B). While the kidney of rats given gamma irradiated Nigella sativa (GRNS), showed desquamation in the cytoplasm of the renal tubules, hemorrhage in the renal corpuscle and lymphocytic infiltration (Fig. 2C). Moreover, the kidney of rats given Propolis (P) alone, showed normal structure (Fig. 2D). 


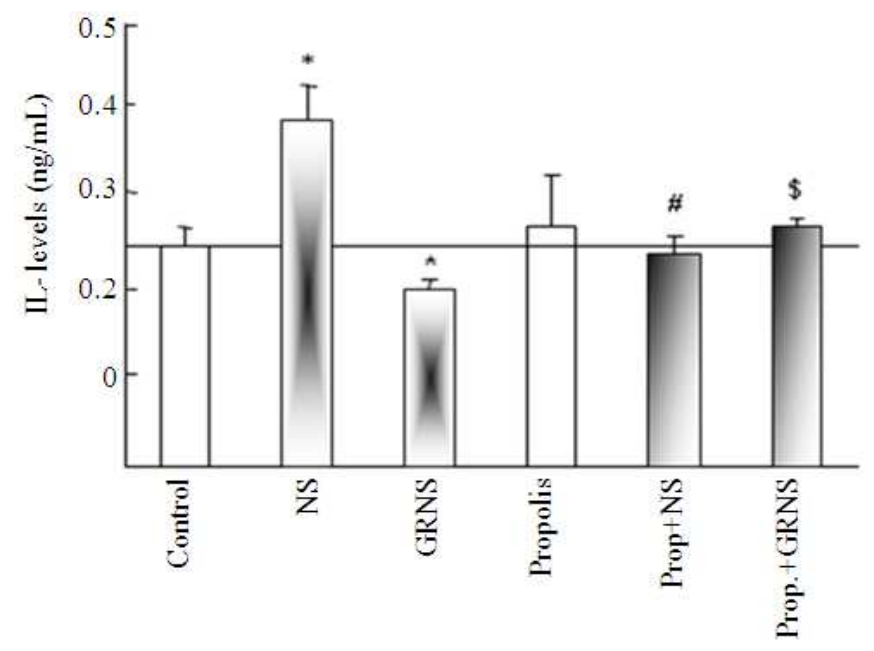

Fig. 5. Protective effects of propolis on NS and GRNS induced changes in IL-10 levels. Wistar rats were NS, GRNS alone or in combination with propolis for 2 weeks. Serum levels of IL-10 were measured using ELISA. Values are means \pm S.E.M for 5 different rats for each experiment. ${ }^{*} \mathrm{p}<0.05 \mathrm{Vs}$ control; $\# \mathrm{p}<0.05$ Vs. NS alone and $\# \mathrm{p}<0.05$ Vs GRNS

While kidney of rats given NS and Propolis (NSP), showed desquamation and vacuolation in the cytoplasm of the renal tubules (Fig. 2E). Finally the kidney of rats given Gamma Irradiated Nigella Sativa and Propolis (GRNSP), showed disappearance of the lymphocytic infiltration and desquamation in the epithelium of the renal tubules (Fig. 2F).

\subsection{Changes in IL-1 $\beta$, TNF- $\alpha$ and IL-10 Levels in Serum of NS, GRNS and Propolis Administration in Wistar Rats}

As seen in Fig. 3 NS, GRNS and propolis significantly $(\mathrm{p}<0.05)$ modulated IL-1 secretion and increased its concentration. Administration of propolis to NS and GRNS did not induce any additive alteration in IL-1 secretion. Unlike IL-1 findings, NS alone modulated $\mathrm{TN}-\alpha$ secretion and GRNS has no effect. Administration of propolis with NS (NSP group) inhibited NS induced alteration in TNF- $\alpha$ (Fig. 4). Parallel to this finding, NS induced IL-10 levels and GRNS inhibited such effect. Addition of propolis to NS (NSP group) inhibited NS induced IL-10 secretion. While propolis administration in GRNSP group normalized the inhibition in IL-10 secretion induced by GRNS alone (Fig. 5).

\section{DISCUSSION}

The current study showed that co-administration of propolis with NS improved alterations occurred by both
NS and GRNS in blood profiles, liver and kidney parameters. Several studies have confirmed the role of flavonoids in the deactivation of the free radicals (Galati and O'Brien, 2004; Kumazawa et al., 2003). But very few data have been published considering the effect of propolis and its polyphenolic compounds in the protection from the whole body irradiation (Dimov et al., 1992). Flavonoids posses' anti-oxidant activity based on ability of direct free radicals scavenging, or stabilizing the ROS by reacting with the reactive compound of the radicals. Because of the high reactivity of the hydroxyl substituents of the flavonoids, radicals are made inactive. Flavonoids can also increase the function of the endogenous anti-oxidant enzyme systems. Furthermore, anti-oxidant effects may be a result of a combination of radical scavenging and an interaction with enzyme functions (Galati and O'Brien, 2004).

NS and GRNS increased IL-1 $\beta$ levels and propolis addition did not induce any additive alteration. This alteration is in away to potentiate the immune response of body to enforce the body defensive mechanism. Regarding alterations occurred on blood, GPT, GOT and Urea levels, propolis is known to have radio-protective effects. The use of flavonoids as potential radio-protectors is of increasing interest because of their high anti-oxidant activity and abundance in the diet (Ritidech et al., 2005). Moreover, propolis is also well known for its medical effects, including anti-inflammatory, anti-viral, immunestimulatory and carcinostatic activities. Many studies reported beneficial properties of propolis in vivo and in vitro (Varanda et al., 1999). 
Regarding interleukin-10 findings, IL-10 is a potent anti-inflammatory cytokine. It inhibits IL-1 $\beta$ and IL-6 production from macrophages (Fiorentino et al., 1991). This cytokine plays a critical role in shaping the immune response by blocking class II major histocompatibility complex expression and decreasing pro-inflammatory cytokine expression (Donnelly et al., 1999; Moore et al., 2001). In our findings we reported that GRNS inhibited IL-10 secretion and co-addition of propolis normalized such inhibition. The stimulation of IL-10 secretion was reported in study of Cole et al. (2010) and is parallel to our findings. NS modulated TNF- $\alpha$ secretion but $\gamma$-irradiated NS inhibited TNF- $\alpha$ secretion and propolis normalized it. Such findings may be attributed the reflex body defensive mechanism to increase the proinflammatory cytokines that initiate body immune response.

As known exposure to ionizing radiation produces reactive oxygen species like hydroxyl radicals, superoxide anions and other oxidant as $\mathrm{H}_{2} \mathrm{O}_{2}$ which cause antioxidant/oxidant balance (Thangasamy et al., 2009). Cytokines play important roles during inflammation and radiation (Ao et al., 2009). The response of body to such radiation involves number of mediators as cytokines produced by macrophages, epithelial cells and fibroblasts (Chen et al., 2002; 2005). Exposure of rats to 10 Gy of $\gamma$-irradiation increased levels of IL- $1 \alpha$ and IL- 6 mRNA expression (Linard et al., 2004). Cytokines are signaling molecules that mediate inflammation and immune response. Those molecules have many cellular functions and affect tissue homeostasis, among those cytokines are IL-1 and TNF- $\alpha$ as inflammatory and IL-10 as anti-inflammatory. Body responses include differentiation of T-helper cells into Th1, Th2 and Th0 cells. As known, Th1 cells involved in cellular immunity while Th2 cells are associated with humeral immunity. Th1 cytokines include IFN- $\alpha$ and TNF- $\alpha$ (Zhang et al., 2011). In our results propolis alleviates the alterations occurred at cellular levels and tissue histopathology. The changes induced by GRNS in blood cells profiles, GPT, GOT and urea levels were normalized by propolis. Also, alterations in kidney and liver histopathology were normalized by administration of propolis. The cytokines normalizing effects of propolis are parallel to histopathological findings and suggest that propolis has anti-oxidant like effects. On the same line of investigations, Bachiega et al. (2012), reported that propolis modulated the immune/inflammatory response. In brief, propolis protected the body from the biohazards in immune-modulatory findings induced by gamma irradiated Nigella sativa.

\section{CONCLUSION}

The present study clarified that administration of propolis is beneficial to prevent alterations induced by $\gamma$ irradiation of Nigella sativa. Moreover, propolis modulates changes in blood cells, liver, kidney, cytokines and histopathology of kidney and liver occurred by NS and gamma irradiated NS.

\section{AKNOWLEDGMENT}

This study was supported in part by a Grant-in-Aid of the dean of Scientific Research (Project number 1804-11433), Faculty of Applied Medical Sciences, Taif University, Kingdom of Saudi Arabia.

\section{REFERENCES}

Ali, B.H. and G. Blunden, 2003. Pharmacological and toxicological properties of Nigella sativa. Phytother. Res., 17: 299-305. PMID: 12722128

Ansorge, S., D. Reinhold and U. Lendeckel, 2003. Propolis and some of its constituents down-regulate DNA synthesis and inflammatory cytokine production but induce TGF-betal production of human immune cells. $Z$ Naturforsch C., 58: 580-589. PMID: 12939048.

Ao, X., L. Zhao, M.A. Davis, D.M. Lubman, T.S. Lawrence and F.M. Kong, 2009. Radiation produces differential changes in cytokine profiles in radiation lung fibrosis sensitive and resistant mice. J. Hematol. Oncol., 2: 6-6. PMID: 19187543

Bachiega, T.F., C.L. Orsatti, A.C. Pagliarone and J.M. Sforcin, 2012. The effects of propolis and its isolated compounds on cytokine production by murine macrophages. Phytother Res., 26: 13081313. PMID: 22275284

Benkovic, V., A.H. Knezevic, N. Orsolic, I. Basic and S. Ramic et al., 2009. Evaluation of radio-protective effects of propolis and its flavonoid constituents: In vitro study on human white blood cells. Phytother Res., 8: 1159-1168. DOI: 10.1002/ptr.2774

Borek, C., 2004. Anti-oxidants and radiation therapy. J. Nutr., 134: 3207S-3209S. PMID: 15514309.

Boskabady, M.H., S. Kiani, P. Jandaghi, T. Ziaei and A. Zarei, 2004. Antitussive effect of Nigella sativa in Guinea pigs. Pak. J. Med. Sci., 3: 224-228.

Chen, Y., J. Williams, I. Ding, E. Hernady and W. Liu, 2002. Radiation pneumonitis and early circulatory cytokine markers. Semin. Radiat. Oncol., 12: 26-33. DOI: $10.1053 /$ srao.2002.31360 
Chen, Y., O. Hyrien, J. Williams, P. Okunieff, T. Smudzin and P. Rubin, 2005. Interleukin (IL)-1A and IL-6: Applications to the predictive diagnostic testing of radiation pneumonitis. Int. J. Radiat. Oncol. Biol. Phys., 62: 260-266. DOI: 10.1016/j.ijrobp.2005.01.041

Cole, N., P.W. Sou, A. Ngo, K.H. Tsang and J.A. Severino et al., 2010. Topical 'Sydney' propolis protects against UV-radiation-induced inflammation, lipid peroxidation and immune suppression in mouse skin. Int. Arch. Allergy Immunol., 152: 87-97. DOI: 10.1159/000265530

Dimov, V., N. Ivanovska, V. Bankova and S. Popov, 1992. Immunomodulatory action of propolis: IV. Prophylactic activity against gram-negative infections and adjuvant effect of the water-soluble derivative. Vaccine, 10, 817-823. DOI: 10.1016/0264-410X(92)90043-J

Donnelly, R.P., H. Dickensheets and D.S. Finbloom, 1999. The interleukin-10 signal transduction pathway and regulation of gene expression in mononuclear phagocytes. J. Interferon Cytokine Res., 19: 563-573. DOI: 10.1089/107999099313695

El-Tahir, K.E., M.M. Ashour and M.M. Al-Harbi, 1993. The respiratory effects of the volatile oil of the black seed (Nigella sativa) in guinea pigs: Elucidation of the mechanism (s) of action. General Pharmacol., 24: 1115-1122. DOI: 10.1016/0306-3623(93)90359-6

Epperly, M.W., C. Sikora, S. DeFilippi, J. Gretton, Q. Zhan and D.W. Kufe et al., 2002. MnSOD inhibits iradiation-induced apoptosis by stabilization of the mitochondrial membrane against the effects of SAP kinase p38 and Jnk1 translocation. Radiat. Res., 157: 568-577. DOI: $\quad 10.1667 / 0033-$ 7587(2002)157[0568:MSDSIR]2.0.CO;2

Epperly, M.W., J.A. Gretton, S.J. DeFilippi, C.A. Sikora and D. Liggitt et al., 2001. Modulation of radiationinduced cytokine elevation associated with esophagitis and esophageal stricture by manganese Superoxide Dismutase-Plasmid/Liposome (SOD-PL) gene therapy. Res., 155: 2-14. DOI: 10.1667/00337587(2001)155[0002:MORICE]2.0.CO;2

Epperly, M.W., J.E. Gretton, M. Bernarding, S. Nie, B. Rasul and J.S. Greenberger, 2003a. Mitochondrial localization of copper/zinc superoxide dismutase $(\mathrm{Cu} / \mathrm{Zn} \mathrm{SOD})$ confers radio-protective functions in vitro and in vivo. Radiat. Res., 160: 568-578. DOI: 10.1667/RR3081
Epperly, M.W., M. Bernarding, J. Gretton, M. Jefferson and S. Nie et al., 2003b. Over expression of the transgene for Manganese Superoxide Dismutase (Mn SOD) in 32D cl 3 cells prevents apoptosis induction by TNF- $\alpha$, IL-3 withdrawal and ionizing iradiation. Exp. Hematol., 31: 465-474. DOI: 10.1016/S0301-472X(03)00041-9

Eric, J.H. and A.J. Giaccia, 2012. Radiobiology for the Radiologist. 7th Edn., Lippincott Williams and Wilkins, Philadelphia, PA., ISBN-10: 1451154186, USA., pp: 576.

Fiorentino, D.F., A. Zlotnik, T.R. Mosmann, M. Howard and A. O'Garra, 1991. IL-10 inhibits cytokine production by activated macrophages. J. Immunol., 147: 3815-3822.PMID: 1940369

Galati, G. and P.J. O’Brien, 2004. Potential toxicity of flavonoids and other dietary phenolics: Significance for their chemopreventive and anticancer properties. Free Rad. Biol. Med., 37: 287-303. PMID: 15223063

Gregory, S.R., N. Piccolo, M.T. Piccolo, M.S. Piccolo and J.P. Heggers, 2002. Abstract Comparison of propolis skin cream to silver sulfadiazine: A naturopathic alternative to antibiotics in treatment of minor burns. J. Altern Complement Med., 8: 77-83. DOI: $10.1089 / 107555302753507203$

Hendee, W.R., 1992. Estimation of radiation risks. BEIR $\mathrm{V}$ and its significance for medicine. JAMA, 268: 620-624. PMID: 1629990

Houghton, P.J., R. Zarka, B. de las Heras and J.R.S. Hoult, 1995. Fixed oil of Nigella sativa and derived thymoquinone inhibit eicosanoid generation in leukocytes and membrane lipid peroxidation. Planta Med., 61: 33-36. DOI: 10.1055/s-2006-957994

Konopacka, M. and J. Rogolinski, 2004. Thiamine prevents X-ray induction of genetic changes in human lymphocytes in vitro. Acta Biochem. Pol., 51: 839-843. PMID: 15448744

Koo, H., J.A. Cury, P.L. Rosalen, G.M. Ambrosano and M. Ikegaki et al., 2002. Effect of a mouthrinse containing selected propolis on 3-day dental plaque accumulation and polysaccharide formation. Caries Res., 36: 445-448. DOI: 10.1159/000066535

Kumazawa, S.H., M. Yoneda, I. Shibata, J. Kanaed, T. Hamasaka and T.S. Nakayama, 2003. Direct evidence for the plant origin of Brazilian propolis by the observation of honeybee behavior and phytochemical analysis. Chem. Pharm. Bull., 51: 740-742. DOI: $10.1248 / \mathrm{cpb} .51 .740$ 
Linard, C., C. Marquette, J. Mathieu, A. Pennequin, D. Clarencon and D. Mathe, 2004. Acute induction of inflammatory cytokine expression after gammairradiation in the rat: Effect of an NF-kappa B inhibitor. Int. J. Radiat. Oncol. Biol. Phys., 58: 427434. DOI: 10.1016/j.ijrobp.2003.09.039

Lowry, O.H., N.J. Rosebrough, A.L. Farr and R.J. Randall, 1951. Protein measurement with the Folin phenol reagent. J. Biol. Chem., 193: 265-275. PMID: 14907713

Moore, K.W., R.D.W. Malefyt, R.L. Coffman and A. O'Garra, 2001. Interleukin-10 and the interleukin-10 receptor. Annu. Rev. Immunol., 19: 683-765.DOI: 10.1146/annurev.immunol.19.1.683

Orsi, R.O., J.M. Sforcin, S.R. Funari, V. Bankova, 2005. Effects of brazilian and bulgarian propolis on bactericidal activity of macrophages against salmonella typhimurium. Int. Immunopharmacol., 5: 359-368. DOI: 10.1016/j.intimp.2004.10.003

Pearce, L.L., M.W. Epperly, J.S. Greenberger, B. Pitt and J. Peterson, 2001. Identification of respiratory complexes I and III as mitochondrial sites of damage following exposure to ionizing radiation and nitric oxide. Nitric Oxide Biol. Chem., 5: 128-136. PMID: 11292362

Ritidech, K.N., M. Tungjai and E.B. Worthon, 2005. Protective effect of apigenin on radiation-induced chromosomal damage in human lymphocytes. Mutat. Res., 585: 96-104. DOI: 10.1016/j.mrgentox.2005.04.003
Salem, M.L, 2005. Immunomodulatory and therapeutic properties of the Nigella sativa L. seed. Int. Immunopharmacol., 5: 1749-1770. PMID: 16275613

Thangasamy, T., P. Jeyakumar, S. Sittadjody, A.G. Joyee and P. Chinnakannu, 2009. L-carnitine mediates protection against DNA damage in lymphocytes of aged rats. Biogerontology, 10: 163-172. PMID: 18629605

Varanda, E.A., R. Monti and D.C. Tavares, 1999. Inhibitory effect of propolis and bee venom on the mutagenicity of some direct-and indirect-acting mutagens. Teratog. Carcinog. Mutag., 19: 403-413. PMID: 10587410

Worthen, D., O. Ghoshen and P. Crooks, 1998. The in vitro anti-tumor activity of some crude and purified components of black seed, Nigella sativa. Anticancer Res., 18: 1527-1533. PMID: 9673365

Wu, J., C. Omene, J. Karkoszka, M. Bosland and J. Eckard et al., 2011. Caffeic Acid Phenethyl Ester (CAPE), derived from a honeybee product propolis, exhibits a diversity of anti-tumor effects in preclinical models of human breast cancer. Cancer Lett., 308: 43-53. DOI: 10.1016/j.canlet.2011.04.012

Zhang, H., L.M. Wu and J. Wu, 2011. Cross-talk between apolipoprotein E and cytokines. Mediators Inflamm. Med. Inflamm., 2011: 949072-949072. PMID: 21772670 